\title{
Measuring low-stress connectivity in terms of bike-accessible jobs and potential bike-to-work trips: A case study evaluating alternative bike route alignments in northern Delaware
}

\author{
Peter G. Furth \\ Northeastern University \\ p.furth@neu.edu
}

\author{
Theja V. V. K. Putta \\ Northeastern University \\ pvvktheja@gmail.com
}

\section{Paul Moser}

Delaware Department of Transportation

Paul.Moser@state.de.us

\begin{abstract}
When road segments with high traffic stress are excluded, the remaining network of low-stress roads and trails can be fragmented, lacking connections between many origin-destination pairs or requiring onerous detour. Low-stress connectivity is a measure of the degree to which origins (for this study, homes) and destinations (jobs) can be connected using only low-stress links and without excessive detour. Revision 2.0 to Level of Traffic Stress criteria is introduced and applied to the road and trail network of northern Delaware. A propensity model is proposed to reflect people's declining willingness to ride a bike with greater trip length and detour, accounting for the impact to health and other benefits of cycling. New connectivity measures are introduced that can be interpreted as the number of bike-accessible jobs and the potential number of bike-to-work trips, powerful measures for evaluating alternatives.

These connectivity measures are applied in a case study evaluating alternative alignments for a bike route between Wilmington and Newark, Delaware's two largest cities, separated by a distance of about $20 \mathrm{~km}$ through a largely suburban landscape. The case study explores the benefits of enhancing alternatives with branches that help connect to population and employment centers. We also find that the connectivity gain from constructing multiple alignments is greater than the sum of connectivity gains from individual alignments, indicating that complementarity between the alternatives, which are spaced roughly 5 $\mathrm{km}$ apart, overshadows any competition between them.
\end{abstract}

\section{Article history:}

Received: February 16, 2017

Received in revised form:

November 7, 2017

Accepted: March 14, 2018

Available online: October 22, 2018

Copyright 2018 Peter G. Furth, Theja V. V. K. Putta \& Paul Moser http://dx.doi.org/10.5198/jtlu.2018.1159

ISSN: 1938-7849 | Licensed under the Creative Commons Attribution - Noncommercial License 4.0

The Journal of Transport and Land Use is the official journal of the World Society for Transport and Land Use (WSTLUR) and is published and sponsored by the University of Minnesota Center for Transportation Studies. 


\section{$1 \quad$ Motivation and research contribution}

Like many other transportation agencies, the Delaware Department of Transportation (DelDOT) is actively investing in new bicycling infrastructure, and needs tools for evaluating investments and comparing alternatives. Bicycling facilities can serve both a recreation and a transportation (utilitarian) function, and in urbanized areas, benefits related to transportation use play an especially important role. This paper describes the development of a method for estimating transportation-related benefits of bicycle network improvements in terms of weighted low-stress connectivity:

- "Low stress" means avoiding road segments in which perceived traffic danger is beyond what most people will willingly tolerate. Mekuria, Furth, and Nixon (2012) defined four levels of traffic stress (LTS), with levels 1 and 2 considered "low-stress," i.e., tolerable to the mainstream population. LTS is based on factors such as traffic speed and degree of separation from motor traffic.

- "Low-stress connectivity" is a measure of the degree to which origins and destinations-in this case study, homes and jobs-are connected using low-stress links and without excessive detour (Mekuria, Furth, \& Nixon, 2012; Furth, Mekuria, \& Nixon, 2016). Often, when high-stress links are excluded, the remaining bicycling network is fragmented, with many origin-destination (OD) pairs not connected, and others connected only using highly circuitous routes.

- "Weighted low-stress connectivity" recognizes that OD pairs are not all equally valuable. Weights should certainly account for the size of the origin and destination, as in Mekuria, Furth, \& Nixon (2012). We introduce here additional weights in the form of a propensity function that reflects people's limited willingness to use a bicycle for long distances and on routes involving a lot of detour. We show how the appropriate weights can transform the connectivity measure into estimates of number of bike-accessible jobs and the number of potential bicycling trips.

- The new methodology is applied in a case study comparing alternative alignments for a bike route between Wilmington and Newark, Delaware's two largest cities. Original methodological contributions include refinements to the low-stress criteria, a propensity model for bike travel, and network-wide measures of number of bike-accessible destinations and potential bike trips. In addition, the case study brings up significant issues related to data and to how alternatives are defined.

\section{Refining level of traffic stress criteria}

Winters, Davidson, Kao, and Teschke (2011) and others have shown that the chief deterrent to riding a bike in North American cities is concerns about the danger or stress from traffic. Several methods have been developed for classifying streets by how comfortable or stressful it is to ride there as a function of traffic characteristics (e.g., traffic speed, volume, number of lanes) and bicycling provisions (e.g., bike lanes), including Bicycle Level of Service (Landis, Vattikuti, \& Brannick, 1997) and Bicycle Compatibility Index (Harkey, Reinfurt, \& Knuiman, 1998). This study uses the Level of Traffic Stress method (Mekuria, Furth, \& Nixon, 2012), which has advantages over previous methods in terms of understandability, data requirements, consistency, and ability to account for intersection effects and protected lane treatments (Furth, Mekuria, \& Nixon, 2016). Its four levels of traffic stress are linked to Geller's popular classification of bicyclists (Geller, 2006; Dill \& McNeil, 2013):

- LTS 1: Suitable for children cyclists. Cyclists are either physically separate from traffic, or face a limited volume of low-speed traffic in which they rarely have to deal with more than one vehicle at a time.

- LTS 2: Limits traffic stress to what the mainstream adult population, called "interested but concerned" by Geller, will tolerate. Either cyclists have their own defensible space (i.e., cars reliably stay out of it), or, if in mixed traffic, they have to deal with multiple vehicles at a time only 
at low speeds and infrequently. They are physically separated from high speed and multilane traffic. The criteria for LTS 2 correspond to a large degree with design criteria for Dutch bicycle route facilities (CROW, 2017).

- LTS 3: A level of traffic stress acceptable to those Geller calls "enthused and confident." Involves frequent but not severe interaction with moderate speed or multilane traffic.

- LTS 4: A level of stress acceptable only to the "strong and fearless." Involves being forced to mix with moderate speed traffic or close proximity to high speed traffic.

An updated set of LTS criteria (Furth, 2017), published as Revision 2.0, was used. Updates were made using input from staff at DelDOT and from Arlington County, Virginia, site of another project using LTS. The main impetus for the changes was the need to respond to traffic situations that were not common in San Jose, California, the city for which the original LTS criteria were created, but common in Delaware and/or Arlington, with the most urgent needs being to reclassify high traffic 2-lane roads with $25 \mathrm{mph}$ speed limits as LTS 3 (not 2), and to more carefully define criteria for rural roads. At the same time, the occasion was used to put the criteria into a more readable format, with LTS in the interior table cells and conditions as row and column heads. The following paragraphs summarize the changes to LTS criteria.

\subsection{Using average daily traffic as an input for some road types}

The biggest change in LTS criteria is that average daily traffic (ADT) has been added as an input for roads in which bikes are in mixed traffic (Table 1). Where ADT is low, cyclists rarely encounter more than one motor vehicle at a time, and passing vehicles tend to grant them ample leeway when passing; however, at higher volumes, cyclists will more frequently experience "triple encounters," when a bicycle and two opposite direction vehicles meet, creating an uncomfortable situation in which there is a sudden need for the vehicle approaching from behind to slow down and follow the bicycle until it's clear to pass (Furth, 2008), or else pass with scant clearance. Higher volumes also mean that cyclists will more frequently encounter multiple vehicles driving in a platoon, reducing the visibility and attention that passing motorists give to a cyclist, and creating prolonged intervals in which the cyclist is constrained and threatened (Furth, 2008).

For 1+1 lane roads (that is, 2-lane, 2-way roads with a marked centerline), the critical ADT that triggers LTS 3 on streets with prevailing speeds of 25 or $30 \mathrm{mph}$ is 1500 . This threshold was based on input from an Arlington planner, who reported that citizens frequently complained of being uncomfortable riding on N. Lexington Street, a 1+1 lane road with parking on one side and ADT between 1500 and 2000. Unlaned 2-way roads have higher ADT thresholds because on such roads motorists tend to ride down the middle and are accustomed to adjusting their lateral position to make space for other road users.

The data burden from adding ADT as a factor is limited in several ways. First, ADT is not a factor on roads with bike lanes or bike paths. Second, if the goal is only to distinguish low-stress (LTS 1 or 2) from high, ADT is not a factor on multilane roads. Third, exact values are not needed, but only the range ADT falls in. In cities we have studied, it is safe to assume that streets classified as "local" have a prevailing speed of $25 \mathrm{mph}$ and no centerline; on such streets, the only ADT information needed is whether ADT exceeds 3000 - something rare for a local street, meaning that analysts need only identify exceptions. In a similar study in Arlington, streets classified as local were assumed to have ADT < 1500 , and 1+1 lane streets classified as arterials were assumed to have ADT > 3000; that left only twolane collectors lacking bike lanes as requiring an ADT check. For all of them, it was possible to estimate $\mathrm{ADT}$ from the County's database of turning movement counts, using a standard factor of 10 to expand a peak-hour volume into $\mathrm{ADT}$. 
Table 1: Level of traffic stress criteria for cycling in mixed traffic (Revision 2.0)

\begin{tabular}{|c|c|c|c|c|c|c|c|c|}
\hline \multirow[b]{2}{*}{$\begin{array}{l}\text { Through Lanes } \\
\text { per Direction }\end{array}$} & \multirow[b]{2}{*}{$\mathrm{ADT}$} & \multicolumn{7}{|c|}{ Prevailing speed (mph) } \\
\hline & & 20 & 25 & 30 & 35 & 40 & 45 & $50+$ \\
\hline \multirow{4}{*}{$\begin{array}{c}\text { Unlaned } \\
\text { (no centerline) } \\
\text { yet two-way }\end{array}$} & $0-750$ & LTS 1 & LTS 1 & LTS 2 & LTS 2 & LTS 3 & LTS 3 & LTS 3 \\
\hline & $751-1500$ & LTS 1 & LTS 1 & LTS 2 & LTS 3 & LTS 3 & LTS 3 & LTS 4 \\
\hline & $1501-3000$ & LTS 2 & LTS 2 & LTS 2 & LTS 3 & LTS 4 & LTS 4 & LTS 4 \\
\hline & $3000+$ & LTS 2 & LTS 3 & LTS 3 & LTS 3 & LTS 4 & LTS 4 & LTS 4 \\
\hline \multirow{4}{*}{1} & $0-750 \mathrm{a}$ & LTS 1 & LTS 1 & LTS 2 & LTS 2 & LTS 3 & LTS 3 & LTS 3 \\
\hline & $751-1500 \mathrm{a}$ & LTS 2 & LTS 2 & LTS 2 & LTS 3 & LTS 3 & LTS 3 & LTS 4 \\
\hline & $1501-3000 \mathrm{a}$ & LTS 2 & LTS 3 & LTS 3 & LTS 3 & LTS 4 & LTS 4 & LTS 4 \\
\hline & $3001+\mathrm{a}$ & LTS 3 & LTS 3 & LTS 3 & LTS 3 & LTS 4 & LTS 4 & LTS 4 \\
\hline \multirow{2}{*}{2} & $0-8000 \mathrm{~b}$ & LTS 3 & LTS 3 & LTS 3 & LTS 3 & LTS 4 & LTS 4 & LTS 4 \\
\hline & $8001+b$ & LTS 3 & LTS 3 & LTS 4 & LTS 4 & LTS 4 & LTS 4 & LTS 4 \\
\hline $3+$ & any $\mathrm{ADT}$ & LTS 3 & LTS 3 & LTS 4 & LTS 4 & LTS 4 & LTS 4 & LTS 4 \\
\hline
\end{tabular}

Notes:

a. Limits shown are for 2-way roads; for one-way roads, limits are two-thirds of those given (e.g., 500 instead of 750)

b. Limits shown are for 2-way roads; for one-way roads, limits are half of those given (i.e., 4000 instead of 8000 )

\subsection{Other significant changes to LTS criteria}

The revised LTS criteria account for a wider range of traffic speeds. $20 \mathrm{mph}$ streets are recognized as having lower LTS than $25 \mathrm{mph}$ streets in many situations, and higher end speed categories have been refined so that rural roads can be better classified.

Revised criteria explicitly provide guidance for how ADT limits apply on one-way streets. On multilane one-way streets, volume thresholds are half those of two-way multilane streets. Essentially one-way multilane roads are treated as if they were half of two-way road with twice the traffic, because on multilane roads, cyclists scarcely interact with opposite direction traffic. However, for single lane one-ways, the same volume thresholds apply as on 1+1 lane streets because in both cases, cyclists face potential stress from all vehicles regardless of direction (recall the triple encounters described earlier).

Revised criteria for riding in bike lanes and shoulders not alongside a parking lane are given in Table 2 . As in the original criteria, ADT is not a factor because bikes have their own space. The main changes are at higher speeds, where bike lane/shoulder width becomes a factor and the speed threshold between LTS 3 and 4 has increased. These changes recognize the popularity among recreational cyclists of high speed rural roads with wide shoulders such as Delaware's state route 1 between Rehoboth Beach and Bethany Beach.

For all bike lanes, whether alongside a parking lane or not, frequent bike lane blockage is no longer treated as an explicit factor; rather, users are now advised to treat such a situation as one in which bikes are in mixed traffic. Apart from this, no changes were made to criteria for bike lanes alongside a parking lane.

Criteria were also developed for unsignalized intersection approaches with right turn lanes. In Delaware, as a highway approaches a junction with a subdivision entrance, it is common for the shoulder to become an auxiliary right turn lane. This situation will be stressful if cyclists are legally required to merge into the travel lane, or if the turn lane geometry allows vehicles to drive in the turn lane at high speeds (a long turn lane and/or a small turn angle). However, those criteria were not applied in the case study due to an absence of data on where such right turn lanes are located. 
Table 2: Level of traffic stress criteria for cycling in bike lanes and shoulders not alongside a parking lane (Revision 2.0)

\begin{tabular}{|c|c|c|c|c|c|c|c|}
\hline \multirow[b]{2}{*}{$\begin{array}{l}\text { Number of thru } \\
\text { lanes per direction }\end{array}$} & \multirow[b]{2}{*}{$\begin{array}{l}\text { Bike lane } \\
\text { width (ft) }\end{array}$} & \multicolumn{5}{|c|}{ Prevailing speed (mph) } & \multirow[b]{2}{*}{$50+$} \\
\hline & & $<25$ & 30 & 35 & 40 & 45 & \\
\hline \multirow{2}{*}{1 or unlaned } & $6+$ & LTS 1 & LTS 2 & LTS 2 & LTS 3 & LTS 3 & LTS 3 \\
\hline & 4 or 5 & LTS 2 & LTS 2 & LTS 2 & LTS 3 & LTS 3 & LTS 4 \\
\hline \multirow{2}{*}{2} & $6+$ & LTS 2 & LTS 2 & LTS 2 & LTS 3 & LTS 3 & LTS 3 \\
\hline & 4 or 5 & LTS 2 & LTS 2 & LTS 2 & LTS 3 & LTS 3 & LTS 4 \\
\hline $3+$ & any & LTS 3 & LTS 3 & LTS 3 & LTS 4 & LTS 4 & LTS 4 \\
\hline
\end{tabular}

\section{Connectivity and effects of distance and detour}

Transportation networks are typically connected, in the sense that if two points $\mathrm{A}$ and $\mathrm{B}$ are on the network, then it is possible to travel from A to B. In most places, low stress bicycling networks stand out as an exception. Because most local streets are low-stress, the low-stress network is large in terms of number of links. However, because road networks are typically configured to prevent through travel on local streets, those low stress streets tend not to form a coherent network. The low-stress network also includes bicycle paths and streets that have been treated with appropriate bicycling facilities, but those provisions are often limited and opportunistic, with the result that the low-stress network is often fragmented.

Mekuria, Furth, and Nixon (2012) proposed that the essential measure of a bicycling network's ability to serve transportation needs is its low-stress connectivity, meaning the degree to which it is possible to travel between origins $(\mathrm{O})$ and destinations $(\mathrm{D})$ using only low-stress routes and without undue detour. With homes as origins and work places as destinations, their measure of gross connectivity was the number of OD pairs that are connected at a given level of traffic stress, subject to limits on overall distance and detour factor:

$$
\begin{gathered}
G C_{k}=\sum_{i} \sum_{j} \delta_{i j k} O_{i} D_{j} \\
\delta_{i j k}=\left\{\begin{array}{c}
1 \text { if }\left(L_{i j k} \leq L_{\text {max }}\right) \text { and }\left(d_{i j k} \leq d_{\max }\right) \\
0 \text { otherwise }
\end{array}\right.
\end{gathered}
$$

where

$G C_{k}=$ gross connectivity at LTS $k$

$L_{i j k}=$ distance from origin $i$ to destination $j$ at LTS $k$

$L_{\max }=$ distance limit

$d_{i j k}=\frac{L_{i j k}}{L_{i j 4}}=$ detour factor for a trip from $i$ to $j$ at LTS $k$

$d_{\max }=$ maximum detour factor

$O_{i}=$ size of origin $i$ (e.g.,population at $\left.i\right)$

$D_{j}=$ size of destination $j$ (e.g.,employment at $j$ ) 


\section{A propensity model to account for distance and detour effects}

In equation 1, the behavioral aversion to long trips and large detour are accounted for through an all-ornothing qualification function $\delta()$ with arbitrary limits $L_{\max }$ and $d_{\max }$. With this formulation, results can be unduly sensitive to the limits chosen - imagine, for example, a large employment center located 5.5 miles from a large population center, and consider how results could change depending on whether $L_{\max }$ was $5 \mathrm{mi}$ or $6 \mathrm{mi}$. Avoiding an arbitrary distance cutoff was especially important for the case study, since the distance from Wilmington to Newark is roughly 13-20 miles depending on the route alternative. Any distance limit less than 20 miles would appear inconsistent with the project objective of connecting the two cities, and any distance limit between 13 and 20 miles would clearly bias the evaluation in favor of alternatives whose length is below the limit. However, counting OD pairs that are 20 miles apart as equally important as OD pairs that are 5 miles apart clearly runs counter to evidence that people are far less likely to ride the longer distance for transportation trips.

Therefore, instead of a binary qualification function, this study used a propensity function that, beyond specified limits, declines with distance and detour. A constant value for short distances is consistent with bike mode share from the Netherlands (CROW, 2017) which is equally high for the distance bins $0-2.5 \mathrm{~km}$ and $2.5-5 \mathrm{~km}$ (about 37\%) and declines for longer distances. Propensity functions that decline with distance are common in the accessibility literature. For bicycling trips, Iacono, Krizek, and El-Geneidy (2010) estimated simple exponential decay propensity functions for various trip purposes. However, their sample was small, and because it was based on bicycle trip length distribution rather than mode share data, it combines the effects of distance on the general trip length distribution as well as bicycle mode share. Lovelace et al. (2017) used a large dataset from the UK to estimate a propensity function giving bicycle mode share as a function of distance. It has a more complex functional form, rising with distance to a peak at $3 \mathrm{~km}$, presumably due to competition with walking, with generally high values in the range $1-6 \mathrm{~km}$. The decline over the range $6-16 \mathrm{~km}$ is very close to an exponential decay which halves propensity every $3.0 \mathrm{mi}(4.8 \mathrm{~km})$.

For this study, we used a propensity function that is constant with distance up to $4 \mathrm{mi}(6.4 \mathrm{~km})$, and then declines exponentially with parameter $0.231 \mathrm{mi}^{-1}$, which halves propensity every $3.0 \mathrm{mi}$ beyond the critical value. This form is consistent with the mode share data reported from the Netherlands and is a reasonable approximation of results from the UK data if competition from walking at very short distances is not considered important from a policy perspective (say, because walking and cycling are both forms of active, non-polluting transportation). Later, we describe a sensitivity test using a critical distance of 2 miles instead of 4 miles.

Intuitively, we find the constant-then-declining model of propensity appealing because of the special properties of bicycle transportation. Bicycle transportation involves not only a time cost, but also physical effort and exposure to outdoors. These latter two factors involve some disutility, but they also confer countervailing benefits. Humans need physical exercise to stay healthy, and being outdoors is pleasurable and contributes to psychological health. However, beyond the time/distance limit at which one's need for daily exercise and exposure to the outdoors is satisfied, the dominant effect of continued exertion and exposure is negative.

In addition, time spent bicycling for transportation substitutes, at least in part, for time that would have been spent traveling by another mode, usually driving or transit. To the extent that time is substituted, the disutility of time required to travel by bicycle can be heavily discounted. If a person's 4-mile commute takes 22 minutes by bike and substitutes for a 15 -minute commute in a car, that person is getting 22 minutes of exercise and fresh air at a net time cost of only 7 minutes.

Our propensity model was also formulated to account for detour, which we define as the fractional increase in trip length beyond the shortest path. Broach, Dill, and Gliebe (2012) found that cyclists will 
detour to find a lower stress bike route; on average, they would increase their trip distance by $16 \%$ for work commute trips, and $26 \%$ for other trips. Because our model limits travel to low-stress routes, the extra distance required to find a low-stress route will be accounted for in a distance-based propensity model. Our propensity model aims to capture two other factors beyond extra distance. One is the substitution effect - the extra distance needed to find a low-stress route does not increase the travel time by competing modes, and so the substitution effect should not apply to detour (that is, travel distance beyond the shortest path) beyond a small level that people normally associate with bicycling. For this purpose, we used $20 \%$ detour as a critical value.

Second, humans are shortest-path seekers, with an innate resistance to paths involving a lot of detour. Often, people won't look for a route that involves what they consider to be an abnormal level of detour, and therefore won't be aware of such routes. Even if they become aware of a circuitous low-stress route to reach their destination, emotionally, many will discount the existence of such an alternative if the level of detour involved seems unnatural. To model this effect, we propose a detour-based propensity that falls linearly from 1 to 0 between two values of detour factor, $d_{2}$ and $d_{3}$, which for the case study took values of 1.333 and 2 , respectively. The idea is that around a detour factor of 1.333 , some people will begin to consider the level of detour unnatural, and that once the detour factor reaches 2.0, it will seem sufficiently unnatural that almost nobody will consider making such a trip by bicycle.

Combining the distance-based propensity with detour-based propensity, our proposed propensity model is a function of $L_{i j k}$ and $L_{i j 4}$ (the trip length at the specified LTS $k$ and the shortest path distance, which is the distance at LTS 4), which implies the detour factor $d_{i j k}=L_{i j k} / L_{i j 4^{*}}$. This model has 5 parameters, $L_{\text {crit }}, d_{1}, d_{2}, d_{3}$, and $\alpha$, and specifies propensity for four ranges of trip length:

$$
\begin{array}{ll}
\text { if } L_{i j k} \leq L_{1}, & p=1 \\
\text { if } L_{1} \leq L_{i j k} \leq L_{2}, & p=e^{-\alpha\left(L_{i j k}-L_{1}\right)} \\
\text { if } L_{2} \leq L_{i j k} \leq L_{3}, & p=e^{-\alpha\left(L_{i j k}-L_{1}\right)} \frac{L_{3}-L_{i j k}}{L_{3}-L_{2}} \\
\text { if } L_{i j k}>L_{3}, & p=0
\end{array}
$$

where

$p=$ propensity to use a bicycle

$L_{1}=\min \left(L_{c r i t} L_{i j 4} * d_{1}\right)$

$L_{2}=L_{i j 4} * d_{2}$

$L_{3}=L_{i j 4} * d_{3}$

Figure 1 illustrates the model for selected values of $\mathrm{L}_{\mathrm{ij} 4}$, the shortest distance from origin to destination. The dotted line is propensity for any value of $L_{i j 4}$ when detour factor is less than 1.2. 


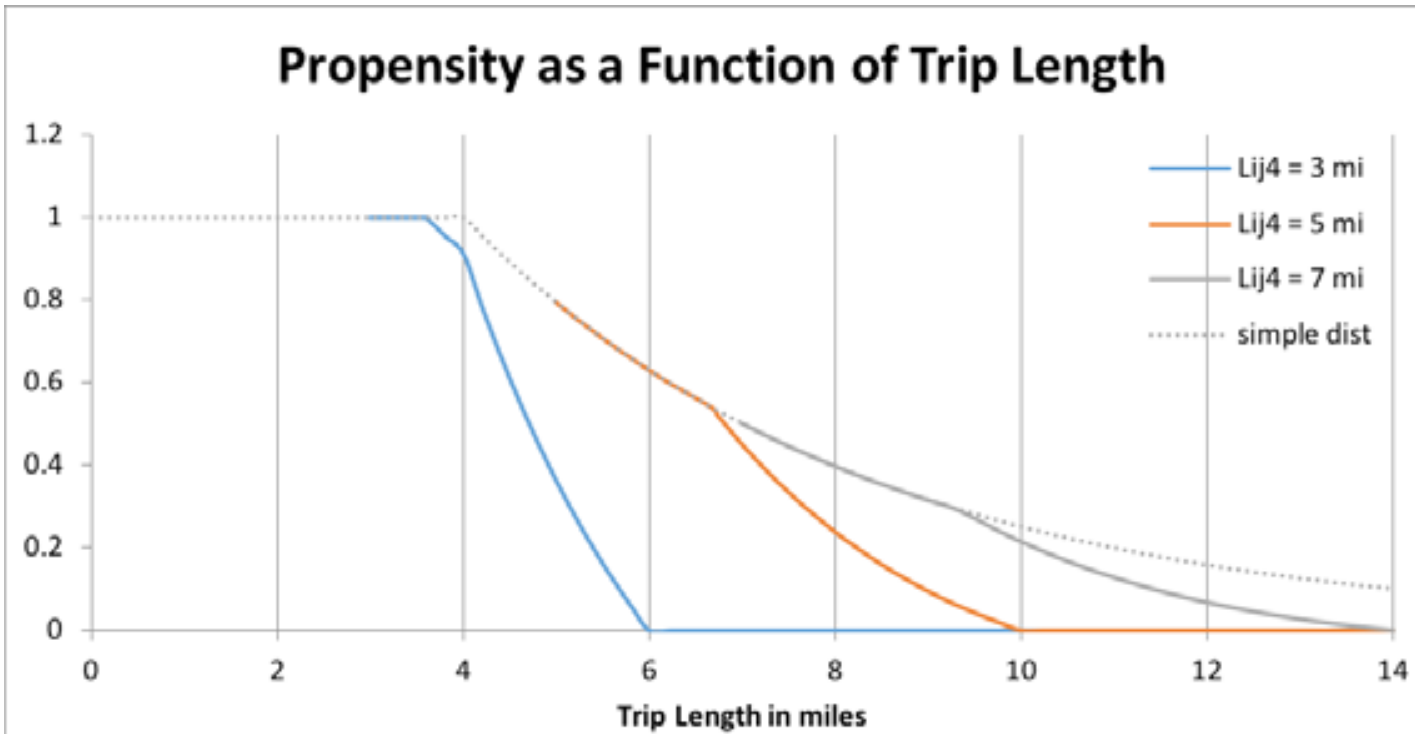

Figure 1: Propensity as a function of trip length. Detour factor is implied (i.e., not shown) as the ratio between trip length and $L_{i j 4}$ "Simple dist" is the propensity that would apply for any value of $L_{i j 4}$ as long as the detour factor $<1.2$.

While the proposed propensity function, like the qualification function used in Mekuria, Furth, and Nixon (2012), still has arbitrarily chosen parameters, its continuous nature ensures that results will not be highly sensitive to chosen parameter values. By allowing long-distance OD pairs to contribute to the benefit measure but with a lower weight, and by allowing that within the population there is range of limits to how much detour a person will accept to follow a low-stress route, the main objections to using an arbitrary distance and detour cutoffs are answered.

\subsection{Number of jobs that are bike-accessible}

Replacing the qualification function $\delta()$ in equation 1 with propensity $p()$ and dividing by total population, the gross connectivity measure becomes

$$
G C_{k}=\frac{\sum \sum p\left(L_{i j k}, L_{i j 4}\right) * O_{i} * D_{j}}{\sum_{i} O_{i}}
$$

Mathematically, GCk is a weighted sum of jobs, with each job weighted by (1) the likelihood that the job-holder lives at various origins, with each member of the population equally likely to hold a given job, and (2) the propensity of using a bike to get to that job from that origin. An interesting interpretation of $G C_{k}$ is

$$
G C_{k}=\text { number of jobs that are bike-accessible at LTS } k
$$

Note that bike accessibility is treated here as a continuous function, so that, for example, 1000 jobs that are 50\% accessible are treated as 500 accessible jobs. 


\subsection{Potential bike-to-work trips}

Rapidly changing social attitudes toward bicycling as a mode of transportation together with gaps in knowledge about bicycling demand make it difficult to make a reliable prediction of how many people will use an improved bicycle network. However, for project evaluation, it is possible to convert $G C_{k}$ into a plausible measure of potential bike-to-work trips by taking advantage of the fact that propensity is, by design, roughly proportional to the likelihood of a person choosing to use a bike. A proportionality factor $\tau$ can be defined:

$\tau=$ bike modal share under ideal connectivity conditions

where "ideal connectivity conditions" are defined as those for which propensity equals 1 - that is, trip length is less than $L_{1}$, and detour factor is less than $d_{2}$. Then, using LTS 2 connectivity (because LTS 2, by design, represents the attitude of the mainstream population), a powerful evaluation measure becomes

$$
\text { potential bike-to-work trips }=\tau^{*} G C_{2}
$$

Clearly, there is a need for future demand modeling research to estimate $\tau$ from behavioral data and to confirm both the form and the parameters of the proposed propensity model. In the meantime, for project evaluation, a transportation agency can choose a value of $\tau$ that is reasonable and consistent with agency goals and aspirations. For this case study, we used the value $\tau=0.2$, reflecting a view that in the long run, if the transportation agency creates low-stress, low-detour routes between people's homes and jobs, $20 \%$ of those with trip length less than $L_{\text {crit }}$ will choose bicycle.

\section{Data sources, cleaning, and integration}

Network data came mainly from DelDOT's county-level road inventory file and its E-911 map file. LTS was calculated using the road inventory file whose attributes include number of lanes, presence of a median, and shoulder width, and whose features include roads and paved shared use paths, which are locally called trails.

However, the road inventory file is not "routable," meaning that roads that appear to intersect may not actually intersect topologically, and therefore cannot be used directly for connectivity analysis. On the other hand, the E-911 file, used for emergency response, is routable. Because there is a one-to-many relationship between the road inventory file and the E-911 file, LTS values calculated in the road inventory file could be pushed to the E-911 file.

Bike lane data, not yet part of DelDOT's road inventory file, was entered manually. Presence of a bike lane was determined from the county planning agency's map, while bike lane attributes were determined through observations in the field or using Google's StreetView. Using DelDOT's sidewalk inventory file, the bike network was augmented with paved paths through parks or campuses, connector paths between shared-use paths and streets, and pedestrian underpasses, overpasses, and median crossings otherwise closed to traffic.

For its pavement management system, DelDOT already had a protocol for assigning ADT measurements made at representative points along a road to the many segments of a road. Segment-level ADT data, held as a separate layer in the road inventory file, was then pushed into the roads layer of the road inventory file. For most roads, this is a simple one-to-one transfer. However, some manipulation was needed for divided roads, which the ADT layer represents as single line features while the road layer 
represents them as dual features.

The network data required a fair amount of data cleaning. Shoulder width data had frequent errors, especially in more urbanized areas, and was spot checked against Google Maps satellite photos. Roads coded as having 4 lanes were checked systematically because some of them, while wide enough for 4 lanes, are striped for only 2 travel lanes with the remainder allowed for parking. Speed limits were spot checked and corrected, although a systematic review would have been impractical. (DelDOT has a separate ongoing project to document speed limit signs and update coded speed limits.)

Population data, by block, comes from the Census Bureau's 2010 census. Employment data, also by block, were taken from the U.S. Census Longitudinal Employment Household Dynamics (LEHD) dataset, based on reports that employers make to the IRS, the federal tax bureau. Figure 2 shows the distribution of population and jobs in northern Delaware. There are some weaknesses in the LEHD data. For businesses with multiple locations, all of their employees may be associated with the location of the business headquarters, a practice that tends to undercount employment at branch or retail locations and to overcount employees at corporate headquarters. DelDOT is currently developing another dataset of employment locations that should not be subject to that weakness, and it would certainly be worthwhile to repeat this case study when that improved dataset becomes available.

To connect census blocks to the road and trail network, each block's population and employment was allocated equally over the intersection nodes lying within a 20-meter buffer of block. An "intersection node" is a node in the street/trail network incident to at least 3 links (i.e., 3 legs). With this model, for a typical rectangular city block, one fourth of its population and employment is allocated to each of the corners. 


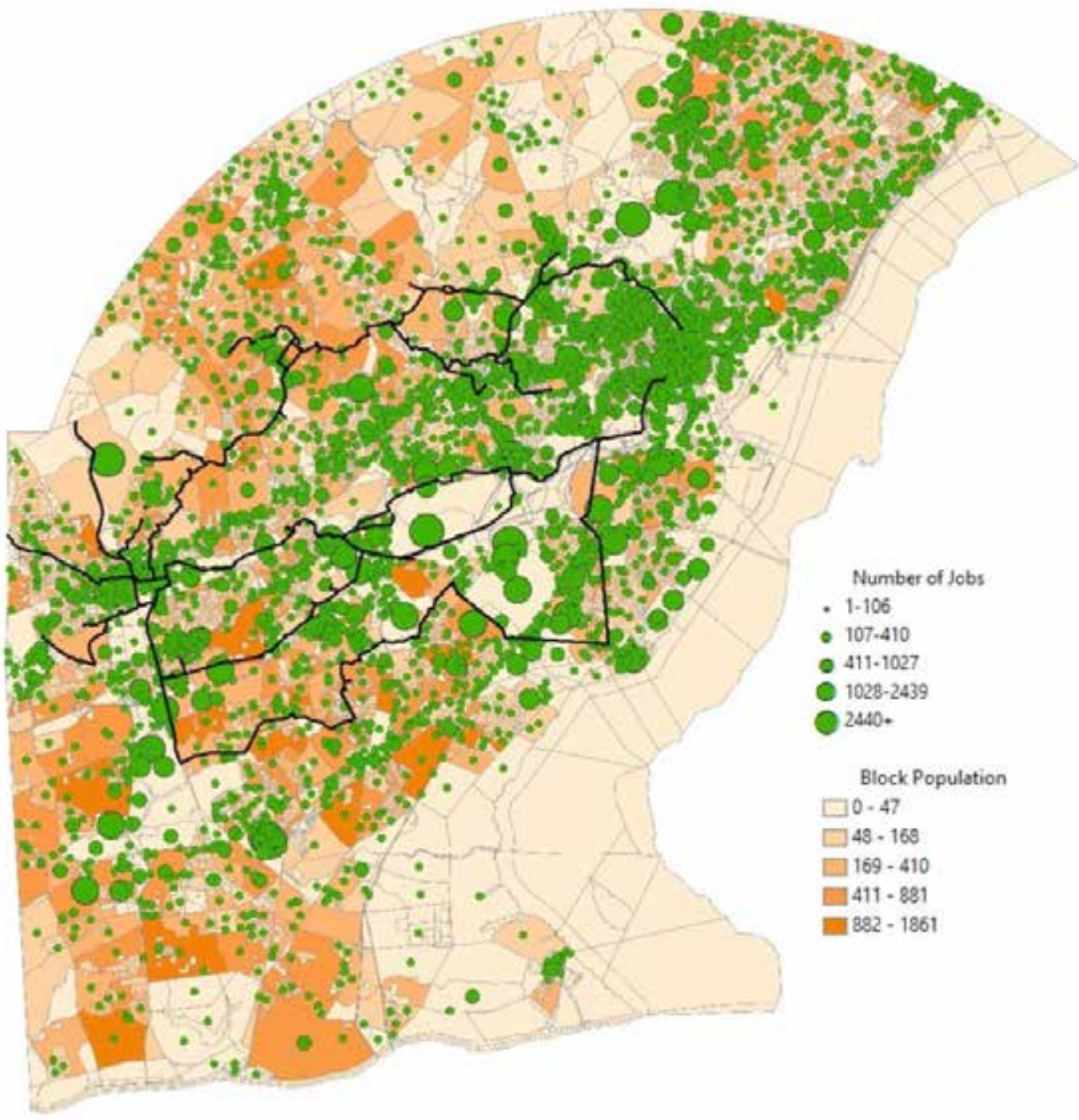

Figure 2: Population and employment distribution in northern Delaware. Black lines indicate the route alignments studied.

\section{$6 \quad$ Alternative greenway alignments}

A 2014 Trail Study commissioned by DelDOT in association with Delaware State Parks and the Wilmington Area Planning Council (Whitman, Requardt \& Associates, 2014) identified links that might be useful as part of a bicycle route connecting Wilmington and Newark. Alignment selection followed a requirement to keep cyclists off-road (a requirement that could be met by sidepaths running alongside roads) and, as much as possible, in a park-like environment in order to make the path attractive for recreational cycling. However, DelDOT recognized that any trail in this well-populated region could also be a vital route for utilitarian travel, and therefore wished to evaluate alignment alternatives in terms of their potential for serving transportation bicycling.

The Trail Study identifies a web of possible route options within which there are four main corridors. We developed a single alignment for each of these corridors, as well as a fifth alternative which enhances the northern alignment by adding branches. The five alternatives are shown in Figure 3. 


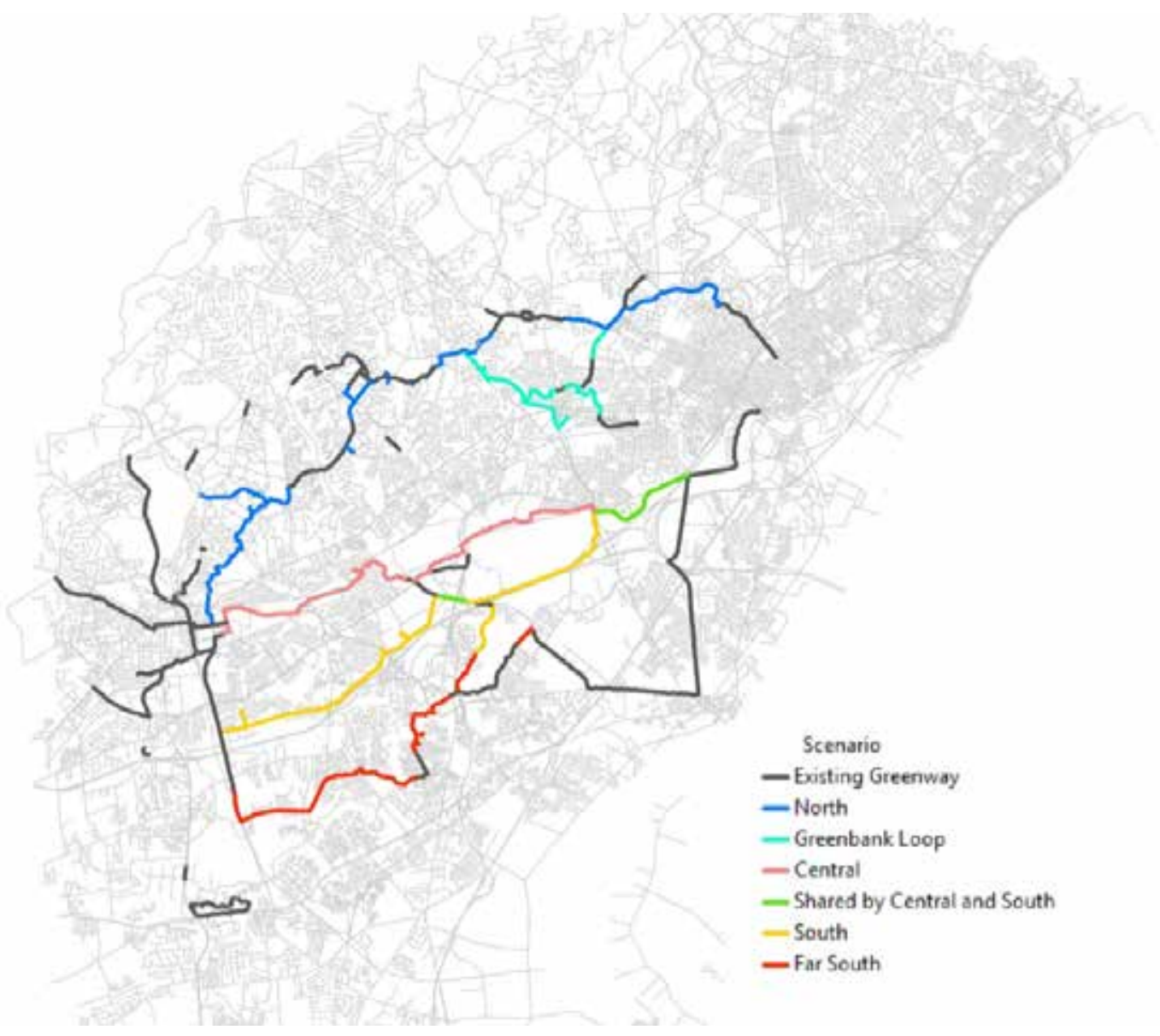

Figure 3: Trail alternatives and constituent segments

For the most part, our alignments use segments identified in the Trail Study. However, in several places we added or substituted segments in keeping with the objective of maximizing low-stress connectivity. Substituted segments aim to improve directness by replacing circuitous routings; added segments improve local connectivity by adding small branches that connect alignments to the local street network. All added and substitute segments appear feasible in terms of right of way and available space. However, they were not required to be off-road; thus, they include local streets with low traffic volumes and low speed.

Considering the connectivity objective, it also makes sense to enhance alignments by including longer branches with potential to create a large connectivity increase at low incremental cost. To test this idea, we created an enhancement for the northern alignment called the Greenbank Loop with branches extending to Price's Corner and Elsmere, areas of population and employment concentration. Because it partially uses existing paths, the connectivity benefit of this enhancement was expected to be high compared to its incremental cost.

Finally, because the alternatives are rather distant from one another from a bicycling perspective, we also created an alternative "Everything but South" that includes all of the routes except the South route, and therefore includes three parallel routes through the study area (North + Greenbank, Central, and Far South). This alternative was included to explore the extent to which the different alignments compete for the same trips. 


\section{$7 \quad$ Results}

Figure 4 shows the number of bike-accessible jobs by level of traffic stress for each of the alternatives. In this analysis the base alternative includes existing paths, paths currently under construction, and local improvements expected to be made in the near future regardless of the alternative chosen, and so it actually represents a considerable improvement from the bicycling network that existed in 2016.

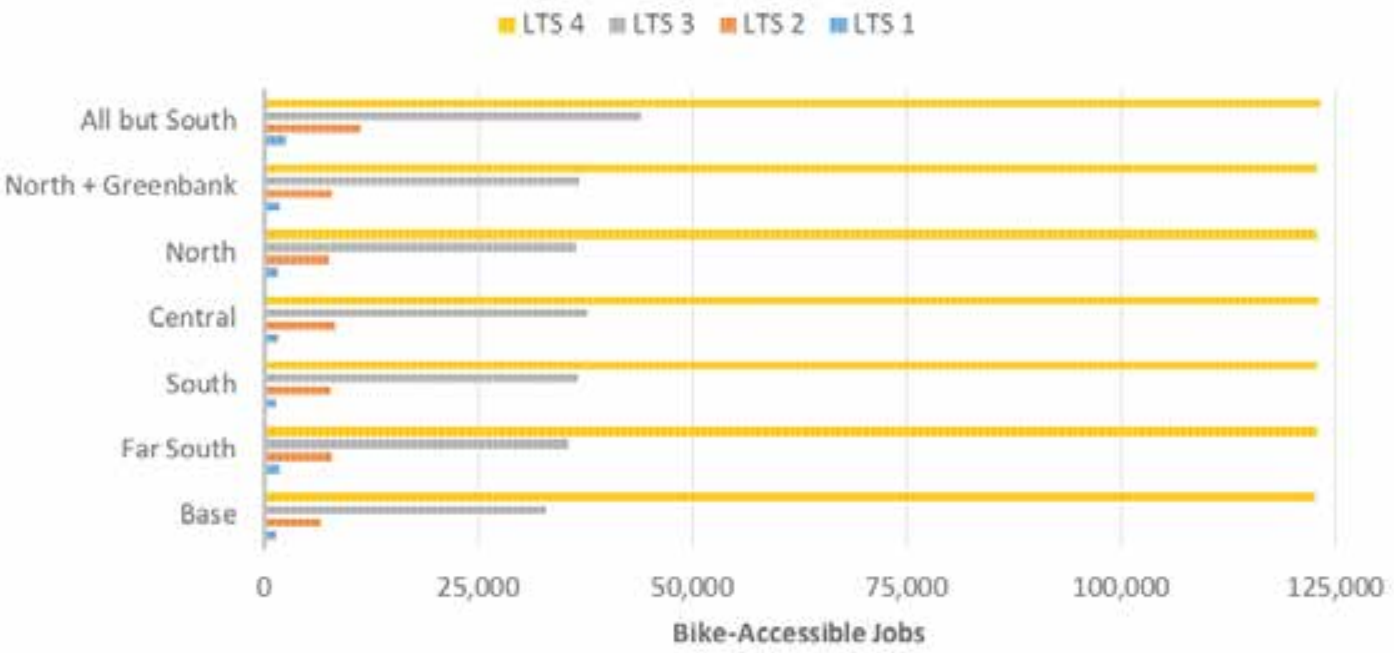

Figure 4: Number of bike-accessible jobs by LTS for each trail alternative

First, one can see that at LTS 4, roughly 122,000 jobs are accessible in every alternative, which is $45 \%$ of the study area's 271,000 jobs. This should be understood as 271,000 jobs that are, on average, $45 \%$ accessible. At LTS 4, lack of accessibility is not due to lack of connectivity, since at LTS 4 all roads except freeways are deemed bikeable, but rather to the long distance between many jobs and homes that result in low propensity for travel by bike. The LTS 4 accessibility of 122,000 can be viewed as an upper limit for bike accessibility, given distances between homes and jobs in the study area.

Second, it is instructive to see how, for any alternative, number of accessible jobs declines sharply at lower levels of traffic stress. In the Base alternative, for example, there is a $73 \%$ dropoff in accessibility from LTS 4 to LTS 3, indicating that in the study area, most home-to-work pairs simply cannot be connected without resorting to LTS 4 roads. At LTS 2 (LTS 1), accessibility is only about 5\% (1\%) of its LTS 4 level, showing how sparse and disconnected is the low-stress network.

Third, a comparison between alternatives shows that the five primary improvement alternatives all offer modest increases in low-stress connectivity. The Central Alternative creates the greatest connectivity improvement at LTS 2, while the Far South and North + Greenbank alternatives show the most improvement at LTS 1.

For greater detail in comparing alternatives, Figure 5 shows each alternative's incremental improvement relative to the base case in terms of potential bike-to-work trips (equation 5). The Central alternative clearly offers the greatest improvement - an addition of 332 potential bike-to-work trips (which is a $25 \%$ improvement compared to the base case). The superior performance of the Central alternative can be attributed to its directness and to its connecting important mid-corridor job and population centers. 


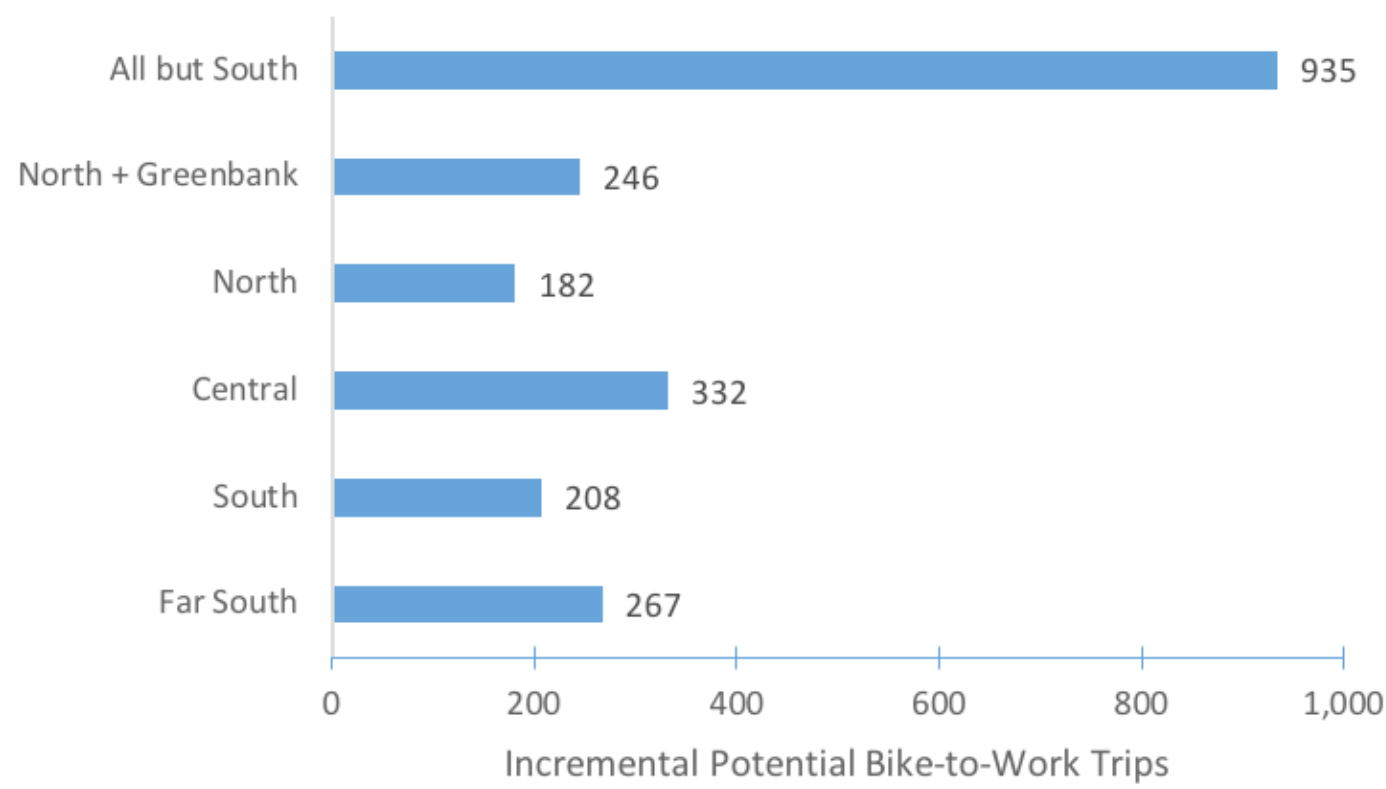

Figure 5: Incremental potential bike-to-work trips for the different alternatives

The worst performing alternative is North, which is considerably less direct and passes through an area with lower population and job density. However, as expected, adding the Greenbank Loop to the North alternative improves connectivity substantially, increasing the number of potential trips added by $33 \%$. This shows the benefit of supplementing a basic alignment with branches that extend to nearby population and job centers, something that should be considered when planning other bike routes.

Finally, we consider the "All but South" alternative. In corridor studies done for other transportation modes, there is typically no thought of providing more than one alignment because they would be competing for the same demand. However, in this study, the distance between alignments is roughly equal to the length of a typical bike trip length, and so the different alignments can be expected to serve distinct populations, removing most of the competition effect.

Indeed, we find that the "All but South" alternative adds 935 potential bike-to-work trips, which is $10 \%$ more than what its three constituent alternatives (North + Greenbank, Central, and Far South) would generate. This indicates not only that the competition effect is small, but also that there is a significant and countervailing complementarity effect, meaning that completion of all three alternatives enables connections are not enabled by any of the alternatives separately, such as between Newark's northern and eastern neighborhoods. Adding additional short branches that would connect the routes mid-corridor would yield a still larger complementarity effect.

While cost estimates for the different alternatives were beyond the scope of this study, Table 3 shows the number of bike-accessible jobs added per mile of new trail, a rough benefit/cost ratio. New trail miles in this analysis do not include local streets that already have LTS 1 or 2, because they need minimal treatment. The Far South alternative looks best, with 242 added accessible jobs per mile of new trail, because while this alternative has the longest path from Wilmington to Newark, it requires the least amount of new trail, only 5.5 miles. Not far behind it is the Central alternative, while the North and South alternatives are far weaker in this metric. 
Table 3: Number of bike-accessible jobs added per mile of new trail

\begin{tabular}{|c|c|c|c|}
\hline Alternative & Added Accessible Jobs & Added Trail (mi) & $\begin{array}{c}\text { Added Accessible Jobs } \\
\text { per Mile }\end{array}$ \\
\hline North & 909 & 9.9 & 92 \\
\hline North + Greenbank & 1,228 & 14.4 & 85 \\
\hline Central & 1,661 & 8.5 & 197 \\
\hline South & 1,041 & 12.6 & 82 \\
\hline Far South & 1,337 & 5.5 & 242 \\
\hline All but South & 4,674 & 30.1 & 155 \\
\hline
\end{tabular}

\section{$8 \quad$ Sensitivity test for critical distance}

The model's sensitivity to the critical distance parameter in the propensity function was tested by repeating the analysis using a critical distance of 2 miles instead of 4 . On an absolute basis, the shorter critical distance lowers average propensity, and thus lowers the number of accessible jobs and potential bike-towork trips by about $30 \%$ for all of the alternatives, including the base case. Differences between alternatives are, of course, similarly affected. However, relative accessibility - meaning the ratio of accessible jobs under any given alternative to what it would be if all roads had low traffic stress - is affected very little. For example, when critical distance changes from 4 miles to 2 miles, the gain in relative accessibility at LTS 2 for the "all but south" alternative changes from $4.7 \%$ to $4.6 \%$.

\section{Conclusion}

Bicycle planners understand the importance of connectivity, and often cite it as a justification for proposed projects; however, until now, they have lacked methods for evaluating the connectivity contribution of a project. This study demonstrates how low-stress connectivity can be quantified and used to evaluate alternatives. A new propensity model solves the problem of arbitrary distance and detour limits that weakened the low-stress connectivity measures previously proposed, and leads to connectivity measures that have intuitive interpretations as number of bike-accessible jobs and potential bike-to-work trips.

The case study showed how using connectivity as an evaluation metric leads to formulating alternatives differently than one might otherwise. This measure rewards alternatives that are more direct, that serve mid-corridor population and employment concentrations, and that include branches that extend a route's reach to population and destination centers. It also shows that when alternative alignments are as distant from one another as those in this study, there can be a strong complementarity effect that justifies implementing not just one of the alignments, but several, and including links that facilitate connections between those alignments.

The emphasis on "low stress" routes, as opposite to "off-road" routes, allows planners to take advantage of existing low-traffic local streets that can reduce cost and improve directness. The low-stress connectivity metric naturally treats all local streets as extensions of the bicycling network, enabling a true end-to-end analysis of trips. 
While this study looked at connecting homes to jobs, one can also consider connectivity between homes and other kinds of destinations including shops, schools, and social activities, as in Lowry et al. (2015). Recognizing these other purposes as well as recreational travel, the number of potential biketo-work trips calculated in this study represents only a fraction of the network's potential demand.

\section{Acknowledgements}

Delaware DOT funded this study. At this time, no decision has yet been made about which trail options to pursue. 


\section{References}

Broach, J., Dill, J., Gliebe, J. (2012). Where do cyclists ride? A route choice model developed with revealed preference GPS data. Transportation Research A, 46, 1730-1740.

CROW. (2017). Design Manual for Bicycle Traffic. Ede, NL: CROW.

Dill, J., McNeil, N. (2013). Four types of cyclists? Examination of typology for better understanding of bicycling behavior and potential. Transportation Research Record: Journal of the Transportation Research Board, 2387, 129-138.

Furth, P. G. (2008). On-road bicycle facilities for children and other 'easy riders': Stress mechanisms and design criteria (paper 08-1074). Proceedings from the Transportation Research Board 87th Annual Meeting, Washington, DC.

Furth, P. G., Mekuria, M. C., \&, Nixon, H. (2016). Network connectivity for low-stress bicycling. Transportation Research Record, 2587, 41-49.

Furth, P. G. (2017). Level of traffic stress criteria for road segments, version 2.0. Retrieved from http:// www.northeastern.edu/peter.furth/criteria-for-level-of-traffic-stress/

Geller, R. (2006). Four types of cyclists. Portland, OR: Portland Bureau of Transportation.

Harkey, D., Reinfurt, D., \& Knuiman, M. (1998). Development of the bicycle compatibility index. Transportation Research Record: Journal of the Transportation Research Board, 1636, 13-20.

Iacono, M., Krizek, K. J., \& El-Geneidy, A. (2010). Measuring non-motorized accessibility: Issues, alternatives, and execution. Journal of Transport Geography, 18(1), 133-140.

Landis, B., Vattikuti, V., \& Brannick, M. (1997). Real-time human perceptions: Toward a bicycle level of service. Transportation Research Record, 1578, 119-126.

Lovelace, R., Goodman, A., Aldred, R., Berkoff, N., Abbas, A., \& Woodcock, J. (2017). The Propensity to Cycle Tool: An open source online system for sustainable transport planning. The Journal of Transport and Land Use, 10(1), 505-528.

Mekuria, M. C., Furth, P. G., \& Nixon, H. (2012). Low-stress bicycling and network connectivity. San Jose, CA: Mineta Transportation Institute, San Jose. Retrieved from http://transweb.sjsu.edu/PDFs/ research/1005-low-stress-bicycling-network-connectivity.pdf

Whitman, Requardt, and Associates (2014). Wilmington-Newark trail study. Dover, DE: Delaware Department of Transportation.

Winters, M., Davidson, G., Kao, D., \& Teschke, K. (2011). Motivators and deterrents of bicycling: Comparing influences on decisions to ride, Transportation, 38, 153-168. 